\title{
Improvements for real-time magnetic equilibrium reconstruction on ASDEX Upgrade
}

\author{
L.Giannone $^{\mathrm{a}}$, R.Fischer ${ }^{\mathrm{a}}$, P.J.McCarthy ${ }^{\mathrm{c}}$, T.Odstrcil ${ }^{\mathrm{a}}$, I.Zammuto ${ }^{\mathrm{a}}$, A.Bock ${ }^{\mathrm{a}}$, G.Conway ${ }^{\mathrm{a}}$, J.C.Fuchs $^{\mathrm{a}}$, A.Gude $^{\mathrm{a}}$, \\ V.Igochine $^{\mathrm{a}}$, A.Kallenbach ${ }^{\mathrm{a}}$, K.Lackner ${ }^{\mathrm{a}}$, M.Maraschek ${ }^{\mathrm{a}}$, C.Rapson ${ }^{\mathrm{a}}$, Q.Ruan ${ }^{\mathrm{b}}$, K.H.Schuhbeck ${ }^{\mathrm{a}}$, W.Suttrop ${ }^{\mathrm{a}}$, \\ L.Wenzel $^{\mathrm{b}}$, ASDEX Upgrade Team ${ }^{\mathrm{a}}$ \\ ${ }^{a}$ Max Planck Institute for Plasma Physics, 85748 Garching, Germany \\ ${ }^{b}$ National Instruments, Austin, TX 78759-3504, Texas, USA \\ ${ }^{c}$ Department of Physics, University College Cork, Cork, Ireland
}

\begin{abstract}
Real-time magnetic equilibria are needed for NTM stabilization and disruption avoidance experiments on ASDEX Upgrade. Five improvements to real-time magnetic equilibrium reconstruction on ASDEX upgrade have been investigated. The aim is to include as many features of the offline magnetic equilibrium reconstruction code in the real-time equilibrium reconstruction code. Firstly, spline current density basis functions with regularization are used in the offline equilibrium reconstruction code, CLISTE [1]. It is now possible to have the same number of spline basis functions in the real-time code. Secondly, in the presence of edge localized modes, (ELM's), it is found to be necessary to include the low pass filter effect of the vacuum vessel on the fast position control coil currents to correctly compensate the magnetic probes for current oscillations in these coils. Thirdly, the introduction of ferromagnetic tiles in ASDEX Upgrade means that a real-time algorithm for including the perturbations of the magnetic equilibrium generated by these tiles is required. A methodology based on tile surface currents is described. Fourthly, during current ramps it was seen that the difference between fitted and measured magnetic measurements in the equilibrium reconstruction were larger than in the constant current phase. External loop voltage measurements and magnetic probe pairs inside and outside the vessel wall were used to measure the vacuum vessel wall resistivity. This is the first step towards including vacuum vessel currents during the plasma current ramp in the real-time equilibrium reconstruction. Fifthly, the introduction of a constraint of the safety factor on the magnetic axis is found to be a helpful method to improve the prediction of the location of rational surfaces for NTM stabilization and disruption avoidance experiments. Soft $\mathrm{X}$-ray tomography is used to assess the quality of the real-time magnetic equilibrium reconstruction using this internal constraint.
\end{abstract}

Key words: real-time, magnetic equilibrium, tokamak control, ferromagnetic tile, soft X-ray tomography

\section{Introduction}

Pre-emptive stabilization of a neoclassical tearing mode, NTM, and disruption avoidance using real-time magnetic equilibria to feedback control a launching mirror for electron cyclotron current drive (ECCD) have been demonstrated in DIII-D, TCV and ASDEX Upgrade $[2,3,4,5,6]$. Real-time magnetic equilibria for the experiments on ASDEX Upgrade are calculated by a Grad-Shafranov solver constrained to fit 40 magnetic probes and 18 flux loop differences. The $33 \times 65$ poloidal flux matrix is available on the reflective memory network with a $2 \mathrm{~ms}$ cycle time.

Real-time magnetic equilibrium reconstruction is an Preprint submitted to Fusion Engineering Design established basis for tokamak control [7, 8, 9]. The procedure for solving the Grad-Shafranov partial differential equation is based on cyclic reduction [10]. A parallelized solver using multiple CPU's and employing discrete sine transforms instead of cyclic reduction has also been developed [11, 12]. A highly parallelized magnetic equilibrium reconstruction code for EAST on a GPU has also been demonstrated [13]. A number of possibilities to add internal constraints to the magnetic equilibrium and the method for constraining the central safety factor, $\mathrm{q}(0)$, to a particular value has been reported [14].

The calculation of eddy currents in a vacuum vessel consisting of several shell sections, where each section is composed of thick plates and bellows made up with

July 27, 2015 
thin sheets, predicts that axially non-symmetric eddy currents will arise [15]. Using the external loop voltage measurements as a simple means for obtaining a vessel current measurement to improve the equilibrium reconstruction on NSTX has been presented [16]. A detailed study has been performed for optimization of magnetic probe arrays inside and outside the vacuum vessel. It was found that these two magnetic probe arrays are essential for equilibrium reconstruction during a plasma current ramp as currents are induced in the vacuum vessel [17].

For a tokamak with a sufficiently elongated crosssection, it is known that external magnetic measurements can be used to determine the total plasma current, the plasma total stored energy and the plasma inductance [18]. It has been shown that soft X-ray tomography can be used to introduce constraints that improve equilibrium reconstruction of current and q profiles in plasmas where the assumption of constant impurity density on a flux surface is valid [19]. It is the aim of this paper to include the features commonly available in offline magnetic equilibrium reconstruction codes in the real-time equilibrium reconstruction code used on ASDEX Upgrade.

Five improvements to the real-time magnetic equilibrium reconstruction code, JANET, on ASDEX Upgrade [5] have been investigated. In Section 2, the idea needed to calculate spline current density basis functions in real-time for the magnetic equilibrium reconstruction is presented. In Section 3, the importance of the low pass filter effect of the vacuum vessel acting on the fast position control coil currents is discussed. The methodology to include the perturbations of the magnetic probe measurements and magnetic equilibrium produced by the presence of ferromagnetic tiles is formulated in Section 4. The steps for using external loop voltage measurements to explicitly include the modification of the magnetic equilibrium due to vacuum vessel currents during the plasma current ramp is explained in Section 5. The introduction of a constraint of the safety factor on the magnetic axis is found to be necessary to predict the location of rational surfaces for NTM stabilization and disruption avoidance experiments on ASDEX Upgrade with sufficient accuracy. In Section 6, soft X-ray tomography is employed to assess the quality of the real-time magnetic equilibrium reconstruction when applying this internal constraint.

\section{Basis functions}

The magnetic equilibrium for a tokamak is described by the Grad-Shafranov equation :

$$
\begin{gathered}
\frac{\partial^{2} \psi}{\partial R^{2}}-\frac{1}{R} \frac{\partial \psi}{\partial R}+\frac{\partial^{2} \psi}{\partial Z^{2}}=-\mu_{0} R j_{\phi}(\psi) \\
j_{\phi}(\psi)=R \frac{\partial p}{\partial \psi}+\mu_{0} \frac{F}{R} \frac{\partial F}{\partial \psi}
\end{gathered}
$$

where $\psi$ is the poloidal flux per radian, $j_{\phi}$ is the current density, and $R, Z$ and $\phi$ are the cylindrical coordinates. The plasma pressure, $p$, and poloidal plasma current, $F$, are functions of $\psi$. The number of current density basis functions for the solver has been increased to 6 splines for the $\mathrm{p}^{\prime}$ terms and 6 splines for the $\mathrm{FF}^{\prime}$ terms. The coefficients of the basis functions ( $\alpha_{i}$ for the $\mathrm{p}^{\prime}$ terms and $\beta_{i}$ for the $\mathrm{FF}^{\prime}$ terms ) giving the best fit to the magnetic probe and flux loop measurements are obtained with a least squares solver using second-order linear regularization. Second-order linear regularization selects the solution with the least curvature in the current density profile [20]. To achieve a cycle time of $2 \mathrm{~ms}$, it was necessary to use look up tables of pre-calculated values as a function of normalised poloidal flux for generating the current density and the integrals for the evaluation of $\mathrm{F}$ on the poloidal flux matrix.

\section{Infinite impulse response filter}

An infinite impulse response filter relates the input signal and output signal of a filter by the difference equation [21]:

$$
y[n]=\frac{1}{a_{0}}\left(\sum_{i=0}^{M} b_{i} x[n-i]-\sum_{j=1}^{N} a_{j} y[n-j]\right)
$$

where $x[n]$ is the input signal, $y[n]$ is the output signal, $a_{i}$ are the feedback filter coefficients and $b_{i}$ are the feedforward filter coefficients. The order of the filter is set by the value of $\mathrm{M}$ and $\mathrm{N}$ and determines the number of past input, $x[n-i]$, and output, $\mathrm{y}[n-j]$, data points used to calculate the most recent output value.

The amplitude and phase response of the magnetic probes to the currents in the poloidal field coils used for fast vertical plasma position control were measured at four frequencies ( 10, 20, 40 and $80 \mathrm{~Hz}$ ). These measurements and the coil currents are the inputs to a commercial software package [22] that calculates the set of filter coefficients defined in Equation 3. These 


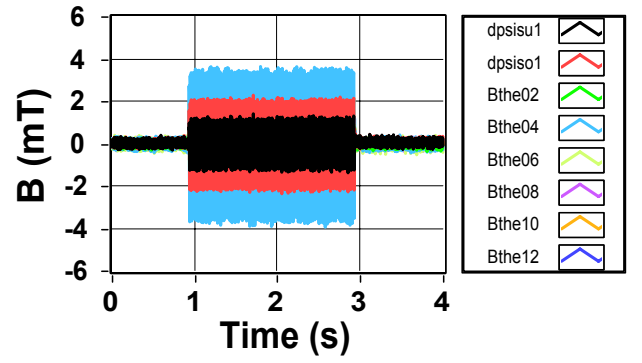

Figure 1: Difference of measured and calculated magnetic probe signal when supplying an $80 \mathrm{~Hz}$ current to the upper fast control coil without compensation for the transfer function due to the vacuum vessel between the control coil and probe. The probe measurements cannot be compensated to the level of $1 \mathrm{mT}$ expected from probe calibration discharges with 2 s flat top current pulses.

coefficients describe the low pass filter effect of the vacuum vessel operating on the coil current. An $80 \mathrm{~Hz}$ current is excited for the time between $0.9 \mathrm{~s}$ and $2.9 \mathrm{~s}$ and the difference between measured and calculated probe measurement for a coil current that does not take into account the low pass filtering effect of the vacuum vessel is shown in Fig. 1. In Fig. 2, the amplitude and phase response of the low pass filter described by the filter coefficients was applied to to the coil current. The difference of measured and calculated probe signal now can be reduced to a value of smaller than $0.5 \mathrm{mT}$. This is a typical value for the difference of measured and fitted probe signals found for current pulses of $2 \mathrm{~s}$ duration in these poloidal field coils. This indicates that it is necessary to apply a low pass filter to the coil current to compensate the measured probe response at this frequency.

In plasma discharges with ELM's, current oscillations in the fast vertical position control coils in the frequency range $30 \mathrm{~Hz}$ to $200 \mathrm{~Hz}$ at the ELM frequency were observed. The magnetic probe measurements now have the contribution due to the oscillating components of the control coil current correctly subtracted. Realtime magnetic equilibria with oscillations in the vertical position resulted when the low pass filtering effect of the vacuum vessel on the control coil current was not taken into account.

\section{Ferromagnetic tiles}

Two rows of ferromagnetic tiles have been added recently to the inner heat shield of ASDEX Upgrade [23]. The tile material, P92, has similar properties to the ferromagnetic material, Eurofer, proposed for DEMO. The real-time calculation of the perturbation of the magnetic probe measurements and the magnetic equilibrium due

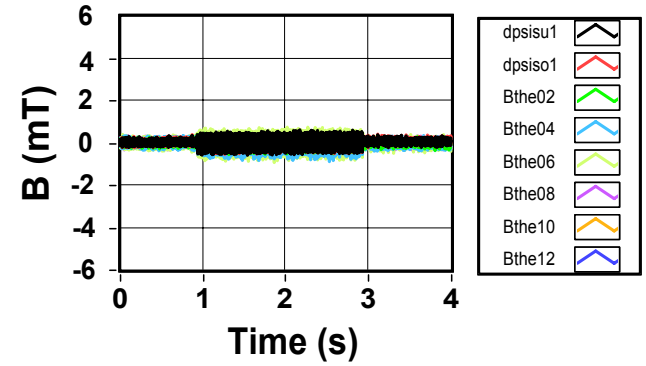

Figure 2: Difference of measured and calculated magnetic probe signal when supplying an $80 \mathrm{~Hz}$ current to the upper fast control coil with compensation for the transfer function due to the vacuum vessel between the control coil and probe. The probe measurements can be now compensated to the level of $1 \mathrm{mT}$ expected from probe calibration discharges with 2 s flat top current pulses.

to these tiles is then necessary. A magnetic probe for poloidal field measurement, with length $13 \mathrm{~cm}$ in the poloidal direction and $4.5 \mathrm{~cm}$ in the radial direction, is situated about $5 \mathrm{~cm}$ away from each ferromagnetic tile row. The tile dimensions of the ferromagnetic tile are $8 \mathrm{~cm}$ in the poloidal direction and $1.4 \mathrm{~cm}$ in the radial direction. The two probes closest to the ferromagnetic tiles are subject to the largest perturbation in measured poloidal magnetic field.

A surface current model of ferromagnetic material in a tokamak is used to calculate these perturbations [24]. The surface current, $\mathrm{I}_{s}(i)$ on the tile element, $\mathrm{i}$, of length, $\mathrm{L}_{i}$, and the tangential component of the external field, $\mathrm{B}_{\tau}^{\text {ext }}(i)$, at the surface of the tile are related by the boundary condition that the tangential components of magnetic field internal and external at each tile surface element are equal. This can be expressed as a matrix equation that can be solved for $\mathrm{I}_{s}(\mathrm{i})[25,26]$ :

$$
B_{\tau}^{e x t}(i)+\sum_{j=1}^{n(j \neq i)} M(i, j) I_{s}(j)=\frac{\mu_{e f f}+1}{\mu_{e f f}-1} \frac{\mu_{o} I_{s}(i)}{2 L_{i}}
$$

where $\mu_{\text {eff }}$ is the effective magnetic permeability and $\mathrm{M}(\mathrm{i}, \mathrm{j})$ is the inductance matrix relating the tangential component of magnetic field at the element $i$ generated by the magnetization current flowing at element $\mathrm{j}$.

The magnitude of the tangential component of magnetic field at the tile surface is known for each poloidal field coil current used in probe calibration discharges without toroidal field and without plasma. Therefore, a $\mu_{\text {eff }}$ can be predetermined for each current flat top. Shown in Fig. 3, are the calculated magnetic probe responses generated by the surface currents of ferromagnetic tiles when exciting current pulses with 2 s dura- 
tion in individual poloidal field coils. The two probes closest to the ferromagnetic tiles have the largest perturbation. A volume average of the calculated probe response is required for those probes close to the ferromagnetic tiles. For real-time calculation, this is reformulated into an average over the mutual inductances between points inside the probe volume and the ferromagnetic tile surface currents.

The small differences of calculated and measured probe response when including the response to the induced surface currents on the ferromagnetic tiles is shown in Fig. 4. At the times in Fig. 3 where the perturbation reaches a steady state value, corresponding to the end of the flat top of the current pulse in the poloidal field coil, the differences for the probes with large perturbations from the ferromagnetic tiles are as small as those probes differences with small perturbations from the ferromagnetic tiles. This indicates that the contribution due to ferromagnetic tiles has been modelled successfully for these probe calibration discharges. The spikes in the differences occur during the rise and fall of the current pulse from the induced current in the passive stabilization loop which decays to zero during the poloidal field coil current pulse flat top. The magnetic probe signal due to the poloidal field coil current is $80 \mathrm{mT}$ for the largest calculated perturbation of $13 \mathrm{mT}$.

For real-time equilibria in plasma discharges with toroidal field, the matrix for calculating the surface currents for 24 elements per tile is computed prior to the discharge, using an experimental look-up table for $\mu_{\text {eff }}$ as a function of $\mathrm{B}$. The external tangential component of magnetic field at each tile element in the matrix equation is calculated at every time step as the sum of the component from the currents in the poloidal field coils and the component due to the plasma current. The matrices for calculating the magnetic probe perturbation and the perturbation of $\psi$ on the grid due to the surface currents are also computed prior to the discharge. The magnitude of perturbations of the magnetic probe measurements is typically a factor of 5 smaller than shown in Fig. 4, as $\mu_{\text {eff } f}$ is much smaller for typical values of $\mathrm{B}_{T}=2.5 \mathrm{~T}$. To determine the current density distribution, $\mathrm{j}_{\phi}(\mathrm{R}, \mathrm{Z})$, flowing in the plasma, the perturbations due to the ferromagnetic tiles and the value of magnetic field due to the currents flowing in the poloidal field coil are subtracted from the magnetic probe measurements. The least squares solver then calculates the coefficients of the spline current basis functions giving the best fit of these corrected measurements.

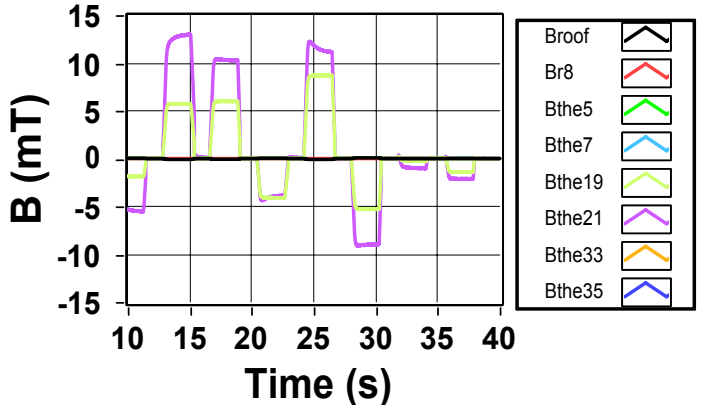

Figure 3: Calculated magnetic probe responses generated by the surface currents of ferromagnetic tiles when exciting current pulses of $2 \mathrm{~s}$ duration in individual poloidal field coils.

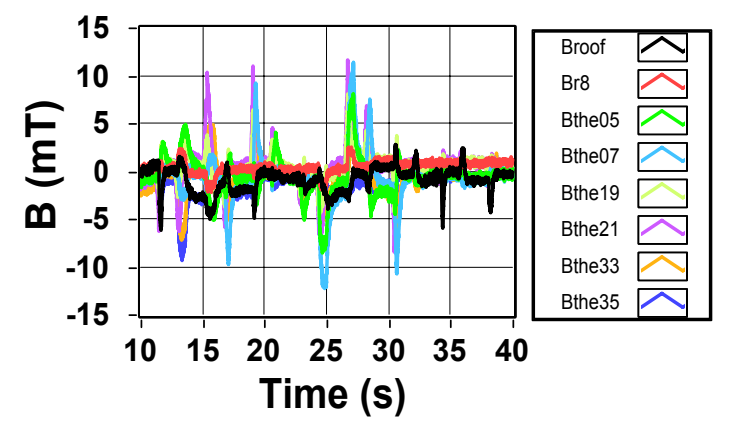

Figure 4: The small differences of calculated and measured probe response when including the response to the induced surface currents on the ferromagnetic tiles is shown. At the times in Fig. 3 where the perturbation reaches a steady state value, corresponding to the end of the flat top of the current pulse in the poloidal field coil, the differences for the probes with large perturbations from the ferromagnetic tiles are as small as those probes differences with small perturbations from the ferromagnetic tiles.

\section{Vessel currents}

This section is devoted to calibrating the vacuum vessel wall resistivity. This would allow a value of current density flowing toroidally in the vessel wall to be calculated straightforwardly from the external loop voltage. This method is chosen as the way to include vessel wall currents in the real-time equilibrium reconstruction rather than using pairs of internal and external probe measurements as constraints. The distributed vessel currents can then be modelled as a number of discrete current filaments. The calculation of the magnetic probe response to a finite number of vacuum vessel current filaments equal to the number of external loop voltage measurements is then simply a matrix multiplication. The alternative would be to increase the number of constraints to additionally fit the internal and external magnetic probe measurement pairs. However, the difficulty of this choice is achieving the required accuracy of com- 
pensation of these magnetic probe measurements due to the currents in the poloidal field coils and the current in the plasma to obtain a reliable measurement of vessel wall current density. A comparison of the advantages and disadvantages of each possible method remains to be carried out.

The time evolution of ohmic heating transformer current used to generate vacuum vessel currents is shown in Fig. 5. The change in magnetic flux also drives a current in the passive stabilization coils of ASDEX Upgrade. The current density in the toroidal direction in the vessel wall can be derived from measurements made by internal and external magnetic probe pairs using the line integral along each of the probes :

$$
\left(B_{i}-B_{e}\right) L=\mu_{o} j w L
$$

where $\mathrm{B}_{i}$ and $\mathrm{B}_{e}$ are the internal and external magnetic probe measurement, $\mathrm{L}$ is the length of the magnetic probe, $\mathrm{w}$ is the width of the vacuum vessel wall and $\mathrm{j}$ is the current density in the vacuum vessel wall. The current densities, calculated for a number of probe pairs, are shown in Fig. 6. The corresponding external loop voltage measurements at various poloidal positions are shown in Fig. 7. A plot of the electric field inferred from the external loop voltages versus the current densities from the closest possible internal and external probe pairs is shown in Fig. 8. The slope of this plot is the resistivity of the vacuum vessel wall and a value of $3 \times 10^{-6} \Omega \mathrm{m}$ is obtained. The value of resistivity for the stainless steel used in the vacuum vessel of thickness $15 \mathrm{~mm}$ is specified as $7.2 \times 10^{-7} \Omega \mathrm{m}$ at $20 \mathrm{C}$. However, there are 8 bellows with $1.2 \mathrm{~mm}$ thickness and approximately $280 \mathrm{~mm}$ length welded between the 8 segments of the vacuum vessel. The bellows are used to increase the toroidal resistance of the vacuum vessel. The effective resistivity is therefore increased above the nominal value for the stainless steel of the vacuum vessel wall. With these values of resistivity, it is now possible to use the external loop voltage measurements to include vacuum vessel currents in a future version of the real-time magnetic equilibrium reconstruction code on ASDEX Upgrade.

\section{Internal constraint}

Without internal constraints for the magnetic equilibrium reconstruction from the Motional Stark Effect, MSE, or polarimeter, it is advantageous to introduce a physically motivated constraint on the safety factor at the magnetic axis, $q(0)$. This relates the coefficient of

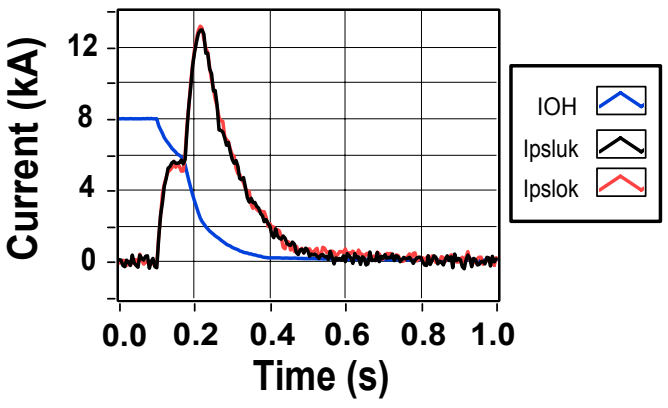

Figure 5: Time evolution of ohmic heating transformer current (IOH) and upper and lower passive stabilization coil current (Ipslok and Ipsluk)

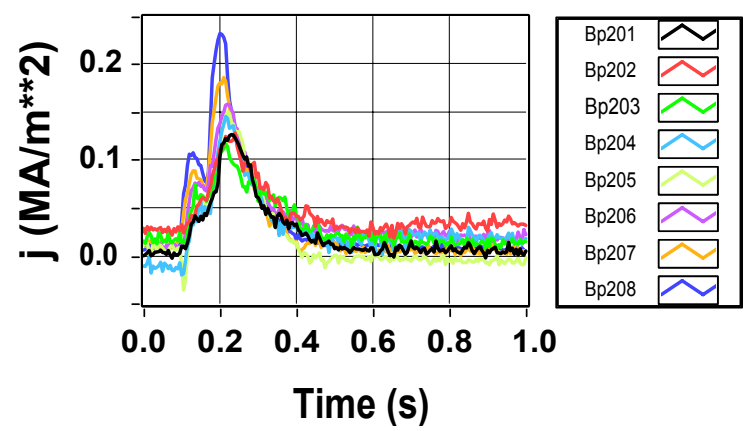

Figure 6: Current densities derived from external and internal magnetic probe pairs using Equation 4.

the spline basis functions at the magnetic axis ( $\alpha_{o}$ and $\beta_{o}$ ) to $\mathrm{q}(0)[27]$ :

$$
\alpha_{o} R_{M}^{2}+\beta_{o}=\frac{2 \pi}{\mu o} \frac{R_{o} B_{o}}{R_{M}} \frac{\kappa^{2}+1}{\kappa} \frac{1}{q(0)}
$$

where $\kappa$ is the plasma elongation, $\mathrm{R}_{M}$ is the radius of the magnetic axis, $\mathrm{R}_{o}$ is the radius of the vacuum vessel axis and $\mathrm{B}_{o}$ is the value of toroidal field at $\mathrm{R}_{o}$. The safety factor profile, $q(r)$, is calculated from the flux surface contour integrals at ten values of normalised poloidal flux. Rational surfaces are located as a function of normalised radius by spline interpolation. For pre-emptive NTM stabilization or disruption avoidance experiments it is necessary to choose a value of $\mathrm{q}(0)$ close to 1 in order to predict the location of the $m / n=3 / 2$ rational surface to within 0.05 of normalised radius. For discharges with an NTM, the location of the rational surface is calculated by ECE correlation analysis at the mode frequency [28]. A feedback controlled launcher mirror is positioned to deposit ECCD on this rational surface.

The soft x-ray diagnostic on ASDEX Upgrade has eight cameras with 208 lines of sight. Data is acquired with a sample frequency up to $2 \mathrm{MHz}$ [29]. The to- 


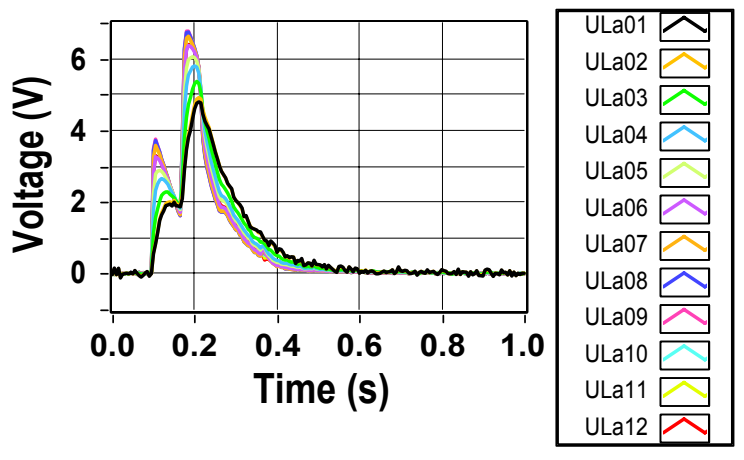

Figure 7: Loop voltages measured outside the vacuum vessel at various poloidal positions

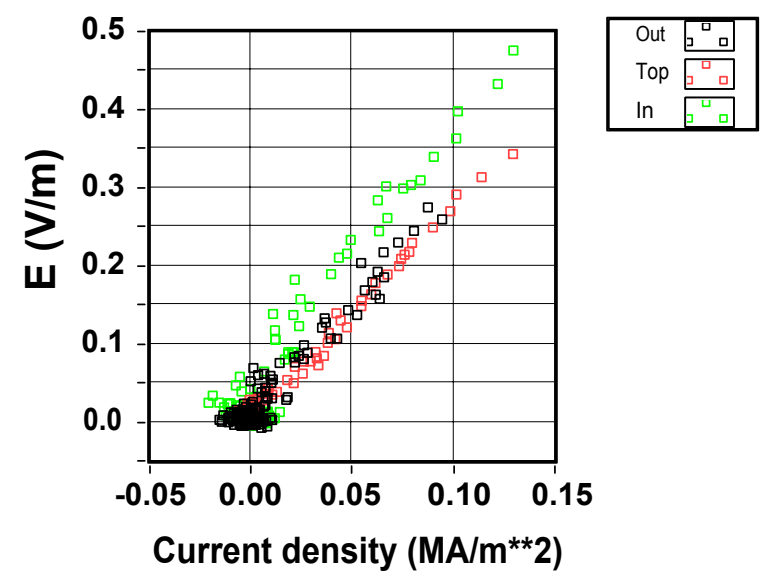

Figure 8: Vessel current density as a function of electric field at the position of the external loop voltage measurements for three poloidal positions. The slope is the resistivity of the vacuum vessel wall at this poloidal position.

mographic reconstruction of the emission power density has been used to assess the quality of magnetic equilibrium construction on DIII-D [19]. It was shown that useful information about the shape of the safety-factor $\mathrm{q}$ profile could be generally determined from the information inferred from surfaces of constant soft X-ray emissivity. However, plasmas with in/out asymmetries in the impurity density profile due to the centrifugal force arising from toroidal rotation of the plasma must be avoided as this can lead to systematic errors in the determination of the magnetic axis.

In Fig. 9, the time evolution of the radial and vertical position of the magnetic axis from real-time (blue) and offline (red) equilibria using only magnetic probe measurements in an ICRH ( $5 \mathrm{MW}$ ) and ECRH ( up to $3 \mathrm{MW}$ ) heated plasma are plotted. This discharge has a plasma current of $1 \mathrm{MA}$, edge safety factor, q95, of 2.6 and a sawtooth frequency of $40 \mathrm{~Hz}$. The emissiv- ity is calculated by tomography on a $80 \times 120$ grid using minimum Fisher regularization [30]. The magnetic axes calculated by the weighted mean of the soft X-ray emission profile with values greater than one third of the maximum (dark green), are also shown. The radial and vertical position of the magnetic axis determined by soft $\mathrm{X}$-ray tomography agrees to within $1 \mathrm{~cm}$ with those values calculated from the real-time magnetic equilibrium. The offline magnetic equilibrium did not impose a $q(0)$ constraint and the radial position of the magnetic axis was systematically larger. A more thorough comparison of magnetic equilibrium reconstruction and soft Xray tomography on ASDEX Upgrade has been started. On TCV, such a comparison identified a systematic difference in the radial position of the magnetic axis that even increased with time due to integrator drift [31].

In Fig. 10, a contour plot of the soft X-ray emissivity before a sawtooth crash is compared to the contours of $\psi$ from real-time (blue) and offline (red) magnetic equilibrium reconstruction. The soft X-ray emissivity is expected to be constant on a flux surface as the lowest possible toroidal rotation is found in discharges with no tangential NBI heating. In this discharge, the central lines of sight have distinct sawtooth crashes and suggest that the $q(0)=1$ constraint assumption is valid. The peak of the soft X-ray emissivity shortly before a sawtooth crash is clearly observed in the tomographic inversion and the contours from soft X-ray tomography are in better agreement with the real-time magnetic equilibrium reconstruction.

Soft X-ray tomography has been employed to assess the quality of the real-time magnetic equilibrium reconstruction. This example indicates that the assumption of a $q(0)=1$ constraint can possibly improve the agreement between the radial position of the magnetic axis from soft X-ray tomography and magnetic equilibrium reconstruction in discharges with sawtooth crashes in the central lines of sight. Internal constraints provided by MSE or polarimeter measurements will be necessary to have real-time magnetic equilibrium reconstruction for NTM stabilization and disruption avoidance experiments on ASDEX Upgrade that do not depend on a $q(0)=1$ internal constraint.

\section{Conclusion}

Five improvements to the real-time equilibrium reconstruction in ASDEX Upgrade have been presented. Real-time correction of the magnetic probe measurements due to the presence of two rows of ferromagnetic tiles calculated by a surface current model has been demonstrated. Probe measurement compensation 


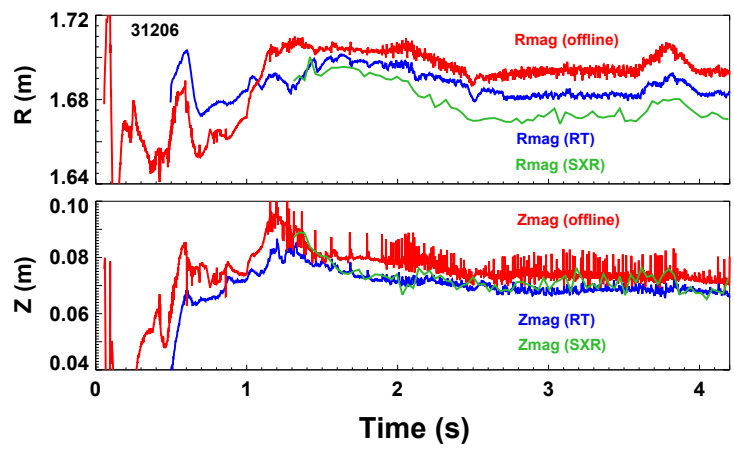

Figure 9: Comparison of the radial and vertical position of the magnetic axis from real-time (blue) and offline (red) magnetic equilibria using only magnetic probe measurements in an ICRH and ECRH heated plasma. The magnetic axes from equilibrium reconstruction from the weighted mean of the upper one third of the soft X-ray emission profile (dark green) is also shown. The use of a $q(0)$ constraint in the real-time equilibrium reconstruction has improved the agreement of the radial and vertical position of the magnetic axis with the positions inferred from soft X-ray tomography.

taking into account the low pass filtering effect of the vacuum vessel on the fast vertical position control coil current was shown to be necessary. Current oscillations in the fast vertical position control coils in the presence of ELM's no longer produce oscillations in the vertical position of the magnetic axis. The equilibrium solver now uses 6 spline functions to represent the $\mathrm{p}^{\prime}$ and $\mathrm{FF}^{\prime}$ terms of the Grad-Shafranov equation and second-order linear regularization to obtain the fit coefficients for the basis current density functions.

External loop voltage measurements and measurements from pairs of magnetic probes inside and outside the vacuum vessel have been used to measure the resistivity of the vacuum vessel as a function of poloidal position. This calibration is the first step towards including vacuum vessel currents, that are present in the plasma current ramp phase of a discharge, in the realtime magnetic equilibrium reconstruction.

The imposition of a $q(0)=1$ constraint was necessary to predict the location of the NTM to within 0.05 of normalised radius. Soft X-ray tomography was employed to assess the quality of the real-time magnetic equilibrium reconstruction using this constraint. In a sawtoothing discharge where the $\mathrm{q}(0)=1$ constraint was valid, there was good agreement between the contours of soft $\mathrm{X}$-ray emissivity and the real-time magnetic equilibrium reconstruction. For the analysed discharge, the radial and vertical position of the magnetic axis determined by soft X-ray tomography agrees to within $1 \mathrm{~cm}$ with those values calculated from the real-time magnetic equilibrium where a $\mathrm{q}(0)=1$ constraint was imposed. The

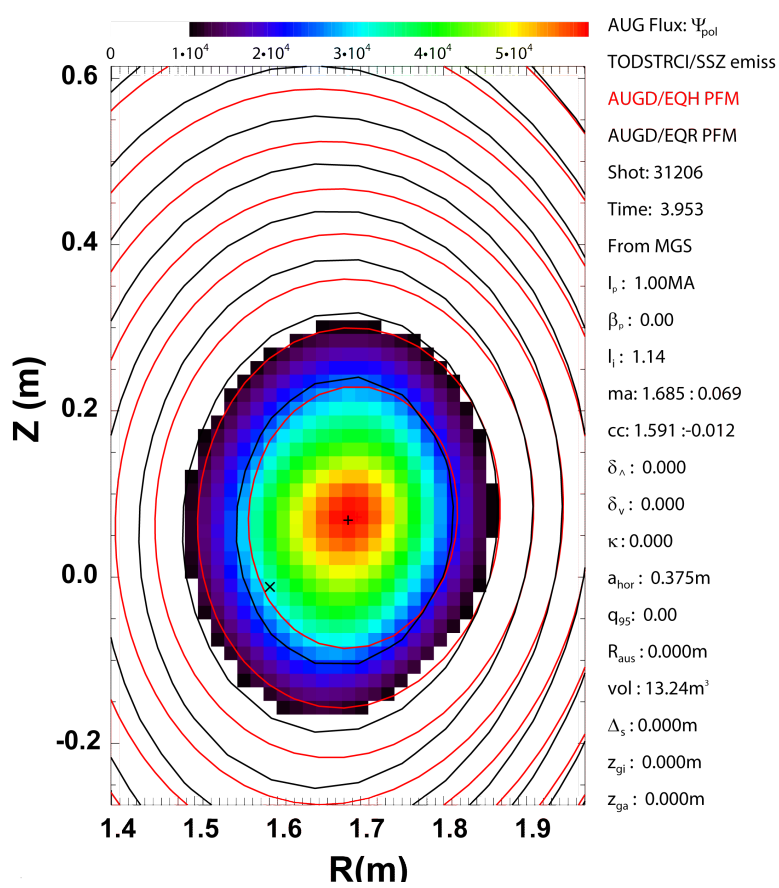

Figure 10: Comparison of soft X-ray emissivity and the real-time (black) and offline (red) magnetic equilibrium in an ICRH and ECRH heated plasma with a peaked emission profile before a sawtooth crash. The contours from soft $X$-ray tomography are in better agreement with the real-time magnetic equilibrium reconstruction.

offline magnetic equilibrium reconstruction did not impose a $\mathrm{q}(0)=1$ constraint and the radial position of the magnetic axis was systematically larger. Internal constraints provided by MSE or polarimeter measurements will be necessary to have real-time magnetic equilibria for NTM stabilization and disruption avoidance experiments on ASDEX Upgrade that do not depend on a $q(0)=1$ internal constraint.

\section{References}

[1] P.J.McCarthy and ASDEX Upgrade Team, Identification of edge-localized moments of the current density profile in a tokamak equilibrium from external magnetic measurements, Plas. Phys. Control. Fusion, 54, 015010 (2012)

[2] R. Prater R.J. La Haye, T.C. Luce, C.C. Petty, E.J. Strait, J.R. Ferron, et al., Stabilization and prevention of the $2 / 1$ neoclassical tearing mode for improved performance in DIII-D, Nucl. Fusion, 47, 1128 (2007).

[3] F. Felici, T.P. Goodman, O. Sauter, G. Canal, S. Coda, B.P. Duval, J.X. Rossel and the TCV Team, Integrated real-time control of MHD instabilities using multi-beam ECRH/ECCD systems on TCV, Nucl. Fusion, 52, 074001 (2012).

[4] M. Reich, L. Barrera, K. Behler et al., NTM stabilization experiments at ASDEX Upgrade, Europhysics Conference Abstracts (Proc. of the 40th EPS Conference on Plasma Physics, Helsinki, Finland) http://ocs.ciemat.es/EPS2011PAP/pdf/P5.102.pdf. 
[5] L. Giannone, M. Reich, M. Maraschek et al., A data acquisition system for real-time magnetic equilibrium reconstruction on ASDEX Upgrade and its application to NTM stabilization experiments, Fusion Eng. Design, 88, 3299 (2013).

[6] B. Esposito, G. Granucci , M. Maraschek et al., Disruption avoidance by means of electron cyclotron waves, Plas. Phys. Control. Fusion, 53, 124035 (2011).

[7] J.R. Ferron, M.L. Walker, L.L. Lao, H.E.St. John, D.A. Humphreys and J.A. Leuer, Real time equilibrium reconstruction for tokamak discharge control, Nucl.Fusion, 36, 1055 (1998).

[8] J. Blum, C. Boulbe and B. Faugeras Reconstruction of the equilibrium of the plasma in a Tokamak and identification of the current density profile in real time, Journal of Computational Physics 231960 (2012)

[9] J.-M. Moret, B.P. Duval, H.B. Le, S. Coda, F. Felici and H. Reimerdes, Tokamak equilibrium reconstruction code LIUQE and its real time implementation, Fusion Eng. Design, 91, 1 (2015).

[10] K. Lackner, Computation of ideal MHD equilibria, Comput. Phys. Commun., 12, 33 (1976).

[11] M.H. Hughes, Solution of Poissons equation in cylindrical coordinates, Comput. Phys. Commun., 2, 157, (1971).

[12] M. Rampp, R. Preuss, R. Fischer, K. Hallatschek, L. Giannone, et al., A parallel GradShafranov solver for real-time control of tokamak plasmas, Fusion Sci. Technol., 62, 409 (2012).

[13] X.N. Yue, B.J. Xiao,Z.P. Luo and Y. Guo, Fast equilibrium reconstruction for tokamak discharge control based on GPU, Plasma Phys. Control. Fusion, 55, 085016 (2013).

[14] L.L. Lao, H.E. St John, Q. Peng et al., MHD equilibrium reconstruction in the DIII-D tokamak, Fusion Sci. Technol., 48, 968 (2005).

[15] T. Kobayashi, Analysis of eddy currents induced in the vacuum vessel of a tokamak device, Japanese Journal of Applied Physics, 18, 2003 (1979).

[16] D.A. Gates, J.E. Menard and R.J. Marsala Vessel eddy current measurement for the National Spherical Torus Experiment, Rev. Sci. Inst., 75, 5090 (2004).

[17] J.A. Romero and J. Svensson, Optimization of out-vessel magnetic diagnostics for plasma boundary reconstruction in tokamaks, Nucl. Fusion, 53, 03300 (2013).

[18] L.L. Lao, H. St.John, R.D. Stambaugh and W. Pfeiffer Separation of $\beta_{p}$ and $l_{i}$ in tokamaks of non-circular cross-section, Nucl. Fusion, 30, 1035 (1990).

[19] J.P. Qian, L.L. Lao, Q.L. Ren et. al, Equilibrium reconstruction of plasma profiles based on soft x-ray imaging in DIII-D, Nucl. Fusion, 49, 025003 (2009).

[20] M. Anton, H. Weisen, M.J. Dutch, W. von der Linden, et. al, X-ray tomography on the TCV tokamak, Plas. Phys. Control. Fusion, 38, 1349 (1996).

[21] W.H. Press, S.A. Teukolsky, W.T. Vetterling and B.P. Flannery, Numerical recipes in C, Cambridge University Press, p561, 1992.

[22] http://zone.ni.com/reference/en-XX/help/372458D01/lvsysid/est_transfer_function_model/

[23] I. Zammuto, L. Giannone, A. Houben, A. Herrmann, A. Kallenbach and the ASDEX Upgrade Team, Long term project in ASDEX upgrade: Implementation of ferritic steel as in vessel wall, Fusion Eng. Design, 96, xxxx (2015).

[24] L.E. Zakharov and V.D. Shafranov, Equilibrium in presence of iron core, Reviews of Plasma Physics, Vol. 11, p264 (1986).

[25] C.V. Atanasiu and L.E. Zakharov, Description of the magnetohydrodynamic equilibrium in iron core transformer tokamaks, Nucl. Fusion, 30, 1027 (1990).

[26] D.P. O' Brien, L.L. Lao, E.R. Solano et al., Equilibrium analysis of iron core tokamaks using a full domain method, Nucl. Fusion, 32, 1351 (1992).

[27] L.L. Lao, J.R. Ferron, R.J. Groebner et al., Equilibrium analysis of current profiles in tokamaks, Nucl. Fusion, 30, 1035 (1990).

[28] M. Reich, A. Bock, M. Maraschek, ASDEX Upgrade Team, NTM localization by correlation of Te and $\mathrm{dB} / \mathrm{dt}$, Fusion Sci. Technol.,, 61, 309 (2012).

[29] V. Igochine, A. Gude and M. Maraschek, Hotlink based Soft X-ray Diagnostic on ASDEX Upgrade, IPP Report No. 1/338, 2010; http://edoc.mpg.de/display.epl?mode=doc: $i d=476537$.

[30] M. Odstrcil, J. Mlynar, T. Odstrcil et al., Modern numerical methods for plasma tomography optimisation, Nuclear Instruments and Methods in Physics Research Section A, 686, 156 (2012).

[31] J.Mlynar, S. Coda, A. Degeling, B.P. Duval, F. Hofmann, T. Goodman, J.B. Lister, X. Llobet and H. Weisen, Investigation of the consistency of magnetic and soft $\mathrm{X}$-ray plasma position measurements on TCV by means of a rapid tomographic inversion algorithm, Plas. Phys. Control. Fusion, 45, 169 (2003).

This work has been carried out within the framework of the EUROfusion Consortium and has received funding from the Euratom research and training programme 2014-2018 under grant agreement No 633053. The views and opinions expressed herein do not necessarily reflect those of the European Commission. 